\title{
Bedside neurophysiological tests can identify neonates with stroke leading to cerebral palsy
}

\section{Nevalainen, Päivi}

2019-05

Nevalainen , P , Metsäranta , M , Toiviainen-Salo, S, Lönnqvist, T, Vanhatalo , S \& Lauronen , L 2019 , ' Bedside neurophysiological tests can identify neonates with stroke leading to cerebral palsy ' , Clinical Neurophysiology , vol. 130 , no. 5 , pp. 759-766 . https://doi.org/10.1016/j.clinph.

http://hdl.handle.net/10138/314539

https://doi.org/10.1016/j.clinph.2019.02.017

publishedVersion

Downloaded from Helda, University of Helsinki institutional repository.

This is an electronic reprint of the original article.

This reprint may differ from the original in pagination and typographic detail.

Please cite the original version. 


\title{
Bedside neurophysiological tests can identify neonates with stroke leading to cerebral palsy
}

\author{
Päivi Nevalainen ${ }^{\mathrm{a}, *}$, Marjo Metsäranta ${ }^{\mathrm{b}}$, Sanna Toiviainen-Salo ${ }^{\mathrm{c}}$, Tuula Lönnqvist ${ }^{\mathrm{d}}$, Sampsa Vanhatalo ${ }^{\mathrm{a}}$, \\ Leena Lauronen ${ }^{\text {a }}$ \\ a Department of Clinical Neurophysiology, Children's Hospital, HUS Medical Imaging Center, University of Helsinki and Helsinki University Hospital (HUH), Helsinki, Finland \\ ${ }^{\mathrm{b}}$ Department of Pediatrics, Children's Hospital, University of Helsinki and HUH, Helsinki, Finland \\ ' Department of Pediatric Radiology, Children's Hospital, HUS Medical Imaging Center, Radiology, University of Helsinki and HUH, Helsinki, Finland \\ ${ }^{\mathrm{d}}$ Department of Child Neurology, Children's Hospital, University of Helsinki and HUH, Helsinki, Finland
}

\section{A R T I C L E I N F O}

Article history:

Accepted 16 February 2019

Available online 15 March 2019

\section{Keywords:}

Neonatal stroke

Electroencephalography (EEG)

Somatosensory evoked potentials (SEP)

Neonatal seizures

Neonatal intensive care

Cerebral palsy $(\mathrm{CP})$

\section{H I G H L I G H T S}

- The unspecific symptoms of neonatal stroke challenge its bedside diagnosis and outcome prediction.

- We show that EEG and SEPs can identify neonates with large middle cerebral artery stroke at bedside.

- SEPs also proved to be accurate in predicting outcome after neonatal stroke.

\begin{abstract}
A B S T R A C T
Objective: The unspecific symptoms of neonatal stroke still challenge its bedside diagnosis. We studied the accuracy of routine electroencephalography (EEG) and simultaneously recorded somatosensory evoked potentials (EEG-SEP) for diagnosis and outcome prediction of neonatal stroke.

Methods: We evaluated EEG and EEG-SEPs from a hospital cohort of 174 near-term neonates with suspected seizures or encephalopathy, 32 of whom were diagnosed with acute ischemic or hemorrhagic stroke in MRI. EEG was scored for background activity and seizures. SEPs were classified as present or absent. Developmental outcome of stroke survivors was evaluated from medical records at 8- to 18months age.

Results: The combination of continuous EEG and uni- or bilaterally absent SEP $(\mathrm{n}=10)$ was exclusively seen in neonates with a middle cerebral artery (MCA) stroke (specificity $100 \%$ ). Moreover, $80 \%$ of the neonates with this finding developed with cerebral palsy. Bilaterally present SEPs did not exclude stroke, but predicted favorable neuromotor outcome in stroke survivors (positive predictive value 95\%).

Conclusions: Absent SEP combined with continuous EEG background in near-term neonates indicates an MCA stroke and a high risk for cerebral palsy.

Significance: EEG-SEP offers a bedside method for diagnostic screening and a reliable prediction of neuromotor outcome in neonates suspected of having a stroke.
\end{abstract}

(c) 2019 International Federation of Clinical Neurophysiology. Published by Elsevier B.V. All rights reserved.

\footnotetext{
Abbreviations: AT, anterior trunk; AIS, arterial ischemic stroke; BE, base excess; $\mathrm{CP}$, cerebral palsy; DM1, distal M1 branch of the MCA; EEG, electroencephalography; HIE, hypoxic-ischemic encephalopathy; ICH, intracerebral hemorrhage; IVH, intraventricular hemorrhage; SEP, somatosensory evoked potential; MRI, magnetic resonance imaging; MCA, middle cerebral artery; NPV, negative predictive value; PVI, periventricular venous infarction; PPV, positive predictive value; PT, posterior trunk; PM1, proximal M1 branch of the MCA.

* Corresponding author at: Department of Clinical Neurophysiology, New Children's Hospital, Helsinki University Hospital, P.O. Box 347, 00029 HUS, Finland.

E-mail address: paivi.nevalainen@hus.fi (P. Nevalainen).
}

\section{Introduction}

Neonatal stroke affects $\sim 1 / 3000$ live births (Estan and Hope, 1997; Lynch and Nelson, 2001; Laugesaar et al., 2007; Takenouchi et al., 2012), and over half of the affected children develop long-term disabilities, including cerebral palsy (Lynch and Nelson, 2001; Lynch, 2009; Rutherford et al., 2012). Acute management of neonatal stroke is essentially supportive (Roach et al., 2008; Kirton and deVeber, 2009; Rutherford et al., 2012; Lehman and Rivkin, 2014). Developing treatment strategies for 
neonates is obstructed by the delay to diagnosis and lack of means to early identification of treatment candidates, i.e. neonates with the most significant neurodevelopmental risks.

Neonatal stroke is typically suspected due to otherwise unexplained motor seizures, but it may also present with recurrent apnea/desaturation, or general signs of encephalopathy (Estan and Hope, 1997; Brouwer et al., 2010; Chabrier et al., 2011; Moharir et al., 2011; Fox et al., 2016; Suppiej et al., 2016). The diagnosis is confirmed with magnetic resonance imaging (MRI) (Lee et al., 2017), which may, however, take time to obtain in the clinical settings. Neonates suspected of having a stroke generally undergo electroencephalography (EEG) at bedside in the acute stage to detect the stroke-related seizures (van Rooij et al., 2010; Low et al., 2014). The diagnostic or prognostic potential of bedside neurophysiological tests has not been assessed, however. A recent study in older children demonstrated that EEG and evoked potentials helped in delineating areas of functional impairment that correlated with anatomical brain lesions (Eytan et al., 2016). Since the neurophysiologically assessable functional impairments arise within minutes after stroke onset (Lee et al., 1997; Penchet et al., 2007), they could offer an immediate bedside marker of stroke, and even neurodevelopmental outcome.

Here, we set out to study which features of simultaneously recorded EEG and somatosensory evoked potentials (SEPs) are useful in the acute phase (1) for diagnosing neonatal stroke and (2) for predicting neuromotor outcome. We hypothesized that a combination of continuous EEG and recurrent, unilateral electrographic seizures would be specific for neonatal stroke (van Rooij et al., 2010; Lynch et al., 2011; Low et al., 2014), and that unilaterally absent SEPs would be specific for stroke and associate with later hemiplegia.

\section{Methods}

\subsection{Participants}

301 consecutive EEG and simultaneous SEP (EEG-SEP) recordings from 270 neonates (postmenstrual age $<46$ weeks) were performed between January 2011 and July 2016 at the department of clinical neurophysiology of the Helsinki University Children's Hospital (Fig. 1). All recordings were done as part of the neonates' clinical diagnostic procedure due to suspicion of seizures or general signs of encephalopathy. After exclusions, 174 neonates were included in the present, retrospective analysis. The exclusion criteria were: gestational age $<34$ weeks (23 neonates), chronological age $>28$ days ( 10 neonates), known brain malformation or known fetal brain injury (10 neonates), multiple organ anomalies (7 neonates), or lack of an MRI (or autopsy) in the neonatal period (46 neonates). Data of some neonates with asphyxia and/or seizures have been included in previous publications (Nevalainen et al., 2017, 2018; Stevenson et al., 2018). The relevant local institutional research review board approved the study protocol. A waiver of consent was given due to the retrospective and observational nature of this study.

\subsection{Magnetic resonance imaging (MRI)}

The brain MRIs were performed with a 1.5 Tesla scanner (Philips Intera Achieva, Philips Medical Systems, Best, The Netherlands) in 169 neonates or with a 3 Tesla scanner (Siemens Magnetom Skyra, Siemens Healthcare $\mathrm{GmbH}$, Erlangen, Germany) in the remaining five. The MRI included at least T1-weighted axial, T2-weighted axial and coronal, and diffusion weighted axial images. We reviewed the MRI reports of all the 174 neonates and identified 32 (11 females) with acute neonatal stroke (according to predefined criteria

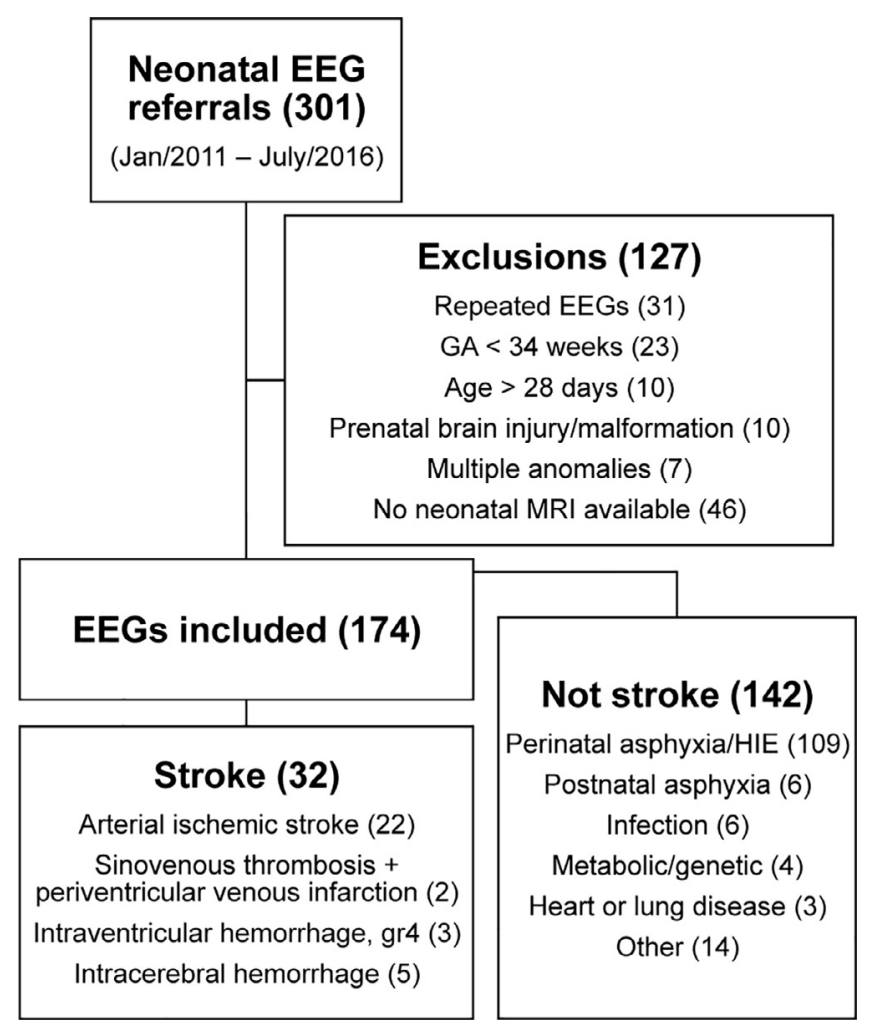

Fig. 1. Flow-chart of study inclusions.

described by Govaert et al., 2009). These included (i) arterial ischemic stroke (AIS), (ii) venous infarction secondary to cerebral sinovenous thrombosis or intraventricular hemorrhage (IVH, grade 4 ), and (iii) intracerebral hemorrhage (ICH). On the contrary, wide spread hypoxic-ischaemic injury or arterial watershed infarction resulting from asphyxia or hypotension were not regarded as stroke. Furthermore, epi- or subdural haematoma and intraventricular haemorrhage (without associated parenchymal injury) were also excluded from the definition of stroke.

An experienced pediatric neuroradiologist (author STS), blinded to the EEG-SEP results and outcome, reviewed the images of those newborns with stroke and further classified the strokes according to predefined criteria (Kirton et al., 2008; Govaert et al., 2009) (Fig. 2). The categories were: (1) Proximal M1 (PM1) = proximal middle cerebral artery (MCA) compromise that includes lateral lenticulostriate arteries, leading to infarction of basal ganglia (globus pallidus, caudate head sparing) and distal MCA territory. (2) Distal M1 (DM1) = MCA compromise distal to the lateral lenticulostriate arteries that spares basal ganglia, while infarcting distal MCA territory. (3) Posterior trunk (PT) = inferior MCA division infarction that includes parietal (posterior-to-central sulcus) and posterior temporal lobes. (4) Anterior trunk (AT) = superior MCA division infarction that includes frontal lobe (anterior-to-central sulcus) and anterior temporal lobe. (5) Other MCA = compromise of a distal branch of the MCA that could not be classified as PT or AT. (6) Other arterial = arterial infarction outside the MCA region. (7) Periventricular venous infarction $(\mathrm{PVI})=$ periventricular white matter medullary venous territory infarction secondary to sinovenous thrombosis. (8) Venous infarction secondary to IVH (grade 4). (9) Intracerebral (lobar) hemorrhage (ICH).

\subsection{EEG-SEP recording and data processing}

The neurophysiological recordings were done with our in-house developed clinical routines where EEG and SEPs are recorded 


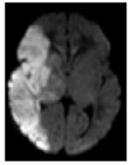

PM1

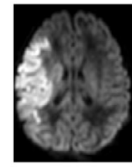

DM1

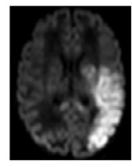

PT



AT

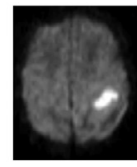

Other MCA

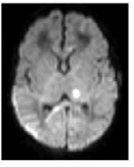

Non-

MCA

arterial

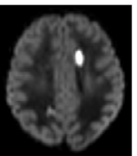

PVI

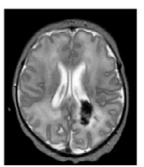

IVH

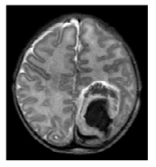

$\mathrm{ICH}$

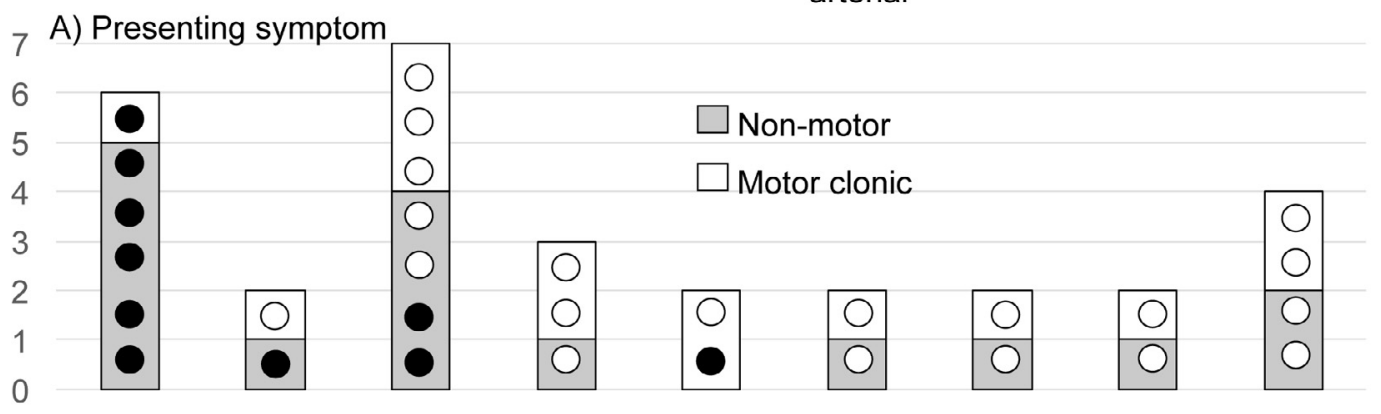

B) SEP

6
5
4
3
2
1

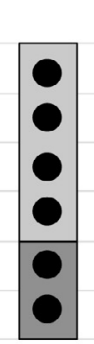

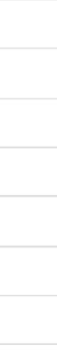
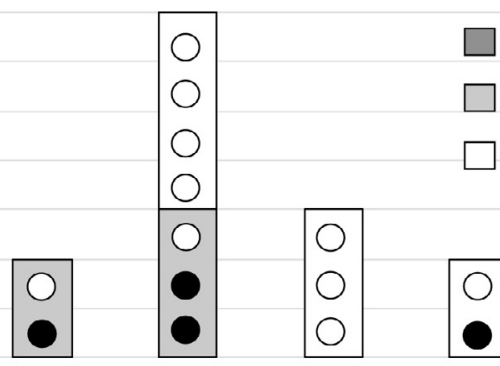

Bilaterally absent

Unilaterally absent

Present
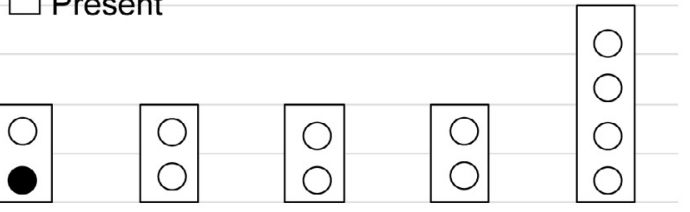

\section{C) EEG}

6
5
4
3
2
1 6
5
4
3
2
1
0
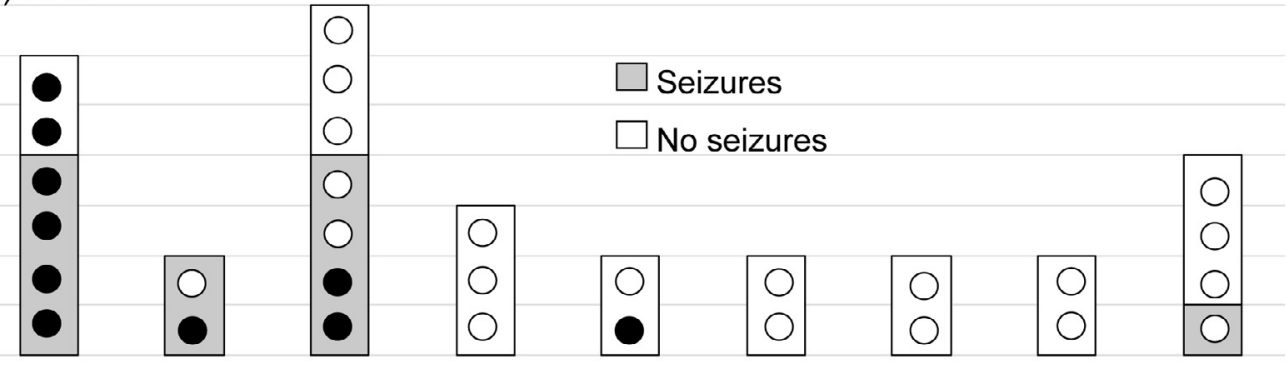

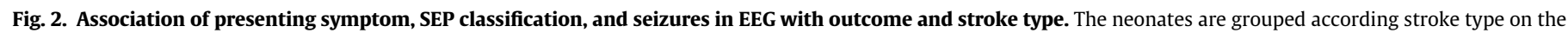

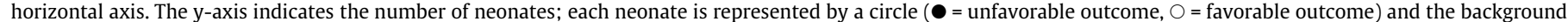

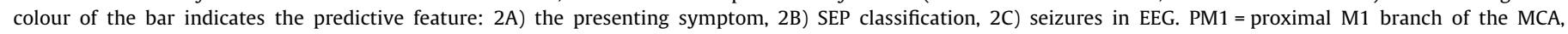

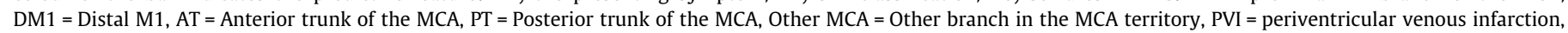
$\mathrm{IVH}=$ intraventricular hemorrhage gr4, ICH = intracerebral hemorrhage.

simultaneously (Nevalainen et al., 2017) using a NicoletOne EEG system (Cardinal Healthcare/Natus, USA; acquisition bandwidth $0.053-500 \mathrm{~Hz}$ ), a sampling frequency of $2000 \mathrm{~Hz}, \mathrm{Cz}$ reference, and 21 channel EEG caps (sintered $\mathrm{Ag} / \mathrm{AgCl}$ electrodes; Waveguard, ANT-Neuro, Germany). For further details of neonatal multichannel EEG recording see http://www.helsinki.fi/science/eeg/videos/ nemo/. An additional electrode was placed on the neck to record the cervical SEP. Polygraphic channels were recorded with disposable silver-silver chloride electrodes (Ambu Blue Sensor, Denmark), including electro-oculogram, electrocardiogram, and submental electromyogram. Respiration was detected with an elastic belt. The median duration of each EEG recording was $75.5 \mathrm{~min}$ (range from 40 to $202 \mathrm{~min}$ ).
The right and left median nerves were stimulated (in separate runs) at the wrist at $0.5-1 \mathrm{~Hz}$ rate using surface electrodes and a portable electrical peripheral nerve stimulator (Micromed Energy Light stimulator; Micromed, Italy). The pulse duration was $0.2 \mathrm{~ms}$, and the stimulation current was individually adjusted to just above the motor threshold. In cases where standard stimulation at the wrist was not possible due to e.g. intra-arterial lines, the median nerve was stimulated at the palm or elbow. Seven neonates only underwent unilateral stimulation (three had Erb's paresis and in four no suitable stimulation site was accessible on one side). The SEPs were averaged offline in BESA ${ }^{\circledR}$ software (BESA $\mathrm{GmbH}$, Germany) for epochs from $-100 \mathrm{~ms}$ to $800 \mathrm{~ms}$ without further filtering. 


\subsection{EEG-SEP classification}

Two neurophysiologists ( $\mathrm{PN}$ and $\mathrm{LL}$ ), blinded to the clinical information (except postmenstrual age), MRI finding, and outcome, performed the neurophysiological analysis according to prespecified criteria. The classification was first done by one neurophysiologist (PN) and the result was compared with the original clinical EEG-SEP report. In case of disagreement with the original report the other neurophysiologist (LL) evaluated the recording and consensus was reached through discussion.

EEG background was classified as continuous (sleep stages identifiable and EEG continuous at least in active sleep / awake state) or discontinuous (sleep stages not identifiable and EEG discontinuous throughout the recording with interburst interval $>10 \mathrm{~s}$ and voltage $<5 \mu \mathrm{V}$ during depression). Seizures were defined as rhythmic, evolving EEG patterns lasting longer than $10 \mathrm{~s}$ and not explained by artefacts. In this paper, the term seizure refers to electrographic seizures with or without coupled clinical signs. Seizures were classified unilateral if they only originated in one hemisphere, and recurrence was defined as at least two seizures during the duration of the EEG recording.

SEPs were classified as present or absent by evaluating the SEP waveforms using a standard longitudinal bipolar montage (with possibility of remontaging) and voltage maps. The cervical response was considered to be present when there was a negativity in the cervical electrode at a latency $<20 \mathrm{~ms}$. Cortical SEP was observed as a salient response peaking within $100 \mathrm{~ms}$ from the stimulation in the contralateral centroparietal area.

\subsection{Outcome}

Outcome of the neonates diagnosed with stroke was evaluated by review of medical records from a follow-up visit to a tertiary center neuropediatric clinic at approximately 1-year age (range: 8-18 months). As the follow-up visit was part of standard care, the neuropediatricians had access to all perinatal test results, including the neonatal EEG-SEP. The outcomes were categorized as favorable (normal motor development), or unfavorable (diagnosis of CP or death).

\subsection{Statistics}

We performed the statistical analysis with IBM SPSS Statistics software v. 23 (SPSS Inc, Chicago, IL, USA). We used the $X^{2}$ test or Fisher's exact test to compare the expected and observed frequencies. For between-groups comparisons of continuous variables we used Student's t-test or Mann-Whitney U test depending on the normality of the data (Kolmogorov-Smirnov-test). For level of statistical significance we chose $p<0.05$. Finally, we calculated the accuracy, sensitivity, specificity, and positive (PPV) and negative predictive values (NPV) with confidence intervals of the prespecified EEG and SEP categories and their combinations to predict unfavorable outcome.

\section{Results}

\subsection{Clinical characteristics and MRI}

MRI revealed a stroke in 32 out of the 174 included neonates (Fig. 1). Of the 32 neonates with stroke, 11 also had a clinical history of asphyxia/hypoxic-ischemic encephalopathy (HIE), which was in most cases mild. Only one of the 11 neonates had clear HIE-related changes in MRI (bilateral ischemic lesion in the basal ganglia/thalamus area) in addition to stroke (subject 2 in Supplemental Table S1). There were no differences in gestational age, postnatal age, gender distribution, birth weight, or the length of the EEG recording between neonates with stroke and those with other etiology (Table 1 ).

Clinical follow-up information was available for 30 of the 32 neonates with stroke (one with ICH and one with IVH were lost to follow-up). Ten of the 30 neonates had an unfavorable outcome at the follow-up: one with concomitant severe HIE died, one developed with severe quadriplegic $\mathrm{CP}$, and eight with hemiplegic $\mathrm{CP}$ (Supplemental Table S1). Compared to neonates with an unfavorable outcome, those with a favorable outcome tended to be more likely to present with clonic motor seizures ( $20 \%$ vs. $60 \%$ vs. respectively; Fisher's exact test, $\mathrm{p}=0.06$, Fig. 2A). Stroke type classified from MRI was significantly associated with outcome ( $X^{2}$ test, $\mathrm{p}<0.01$, Fig. 2).

\subsection{EEG and SEPs in diagnosing stroke}

Table 2 shows the accuracy, sensitivity, specificity, PPV, and NPV of the EEG and SEP features for identifying the neonates with stroke from the full cohort. EEG background was always continuous in neonates that had stroke without concomitant HIE/asphyxia. Seizures were more common in neonates with stroke than the other neonates of the cohort $\left(X^{2}\right.$ test, $\left.\mathrm{p}<0.001\right)$. Particularly, observing recurrent unilateral seizures had a high PPV for stroke (77\%). The PPV was still improved when combining the presence of continuous EEG background to observing recurrent unilateral seizures (83\%). The sensitivity of this combination was only $31 \%$, however.

Absent SEPs (uni- or bilaterally) were more common in neonates with stroke than the other neonates of the cohort $\left(X^{2}\right.$ test $\mathrm{p}=0.03$ ) and particularly unilaterally absent SEPs were strongly associated with stroke (Fisher's exact test, $\mathrm{p}<0.001$ ). Of the 11 neonates with unilaterally absent SEP nine had an MCA infarction (same hemisphere as the absent SEP), whereas the other two had HIE without stroke. Of the 24 neonates with bilaterally absent SEPs only one had bilateral MCA stroke, whereas another one had an MCA stroke in addition to severe HIE, 21 had severe HIE alone, and one had a severe neonatal epileptic encephalopathy (later diagnosed with KCNQ2-mutation).

Combining the SEP result to EEG background classification made diagnostic discrimination even more specific: All ten neonates with stroke-related absent SEP had continuous EEG background, while all the other 25 neonates with absent SEP but no stroke had discontinuous EEG. Consequently, the combination of absent SEP (in one or both hemispheres) and continuous EEG background had a PPV of $100 \%$ for stroke. Though the overall sensitivity of this combination for stroke was only $31 \%$, for stroke with unfavorable outcome the sensitivity was $80 \%$. Thus, the combination found eight out of the ten neonates with stroke and unfavorable outcome (Fig. 3). The other two with stroke and unfavorable out-

Table 1

Clinical characteristics of neonates with stroke and other etiology. There were no statistical differences between the groups in any of the listed characteristics ( $X^{2}$ test, Student's t-test or Mann-Whitney $U$ test depending on the variable type and normality).

\begin{tabular}{lll}
\hline Baseline characteristics & Stroke (32) & Other (142) \\
\hline Females & $10(31 \%)$ & $65(46 \%)$ \\
Birth weight [g] & $3240[530]$ & $3340[590]$ \\
GA [weeks] & $39.7\{3.1\}$ & $40.0\{2.8\}$ \\
PMA at EEG-SEP [weeks] & $40.1\{3.1\}$ & $40.6\{2.7\}$ \\
Postnatal age at EEG-SEP [d] & $3\{3\}$ & $4\{4\}$ \\
EEG duration [min] & $71.5\{34\}$ & $75.5\{31\}$ \\
Time between EEG-SEP and MRI [d] & $1\{2\}$ & $1\{4\}$
\end{tabular}

Data shown as $\mathrm{n}(\%)$, median $\{\mathrm{IQR}\}$, or mean [SD]. GA= gestational age, PMA $=$ postmenstrual age. 
Table 2

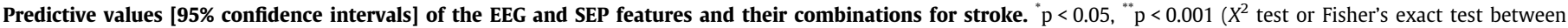
neonates with stroke and those without). NPV = negative predictive value, $\mathrm{PPV}=$ positive predictive value, SEP- $=\mathrm{SEP}$ absent uni- or bilaterally, $\mathrm{SZ}=\mathrm{seizures}$.

\begin{tabular}{|c|c|c|c|c|c|c|c|}
\hline \multirow[t]{2}{*}{ EEG/SEP } & \multicolumn{2}{|l|}{ Stroke } & \multirow[t]{2}{*}{ Accuracy } & \multirow[t]{2}{*}{ Specificity } & \multirow[t]{2}{*}{ Sensitivity } & \multirow[t]{2}{*}{ PPV } & \multirow[t]{2}{*}{ NPV } \\
\hline & No (142) & Yes (32) & & & & & \\
\hline $\mathrm{Sz}^{* *}$ & $13 \%(19)$ & $41 \%(13)$ & $78[71-84]$ & $87[80-92]$ & $41[24-59]$ & $41[27-55]$ & $87[83-90]$ \\
\hline Recurrent unilateral sz & $2 \%(3)$ & $31 \%(10)$ & $86[80-90]$ & $98[94-100]$ & $31[16-50]$ & $77[49-92]$ & $86[83-89]$ \\
\hline Continuous background \& recurrent unilateral sz ${ }^{* *}$ & $1 \%(2)$ & $31 \%(10)$ & $86[80-91]$ & 99 [95-100] & $31[16-50]$ & $83[54-96]$ & 86 [83-89] \\
\hline $\mathrm{SEP}-^{*}$ & $17 \%(24)$ & $34 \%(11)$ & $74[67-80]$ & 83 [76-89] & 34 [19-53] & $31[20-46]$ & $85[81-88]$ \\
\hline Continuous background \& SEP- ${ }^{* *}$ & $0 \%(0)$ & $31 \%(10)$ & 87 [81-92] & $100[97-100]$ & $31[16-50]$ & $100[-]$ & 87 [84-89] \\
\hline
\end{tabular}

come that were missed by this combination were one with discontinuous EEG probably caused by a concomitant severe HIE, and one with normal SEPs but diagnosis of mild hemiplegia at follow-up.

\subsection{EEG and SEPs in predicting outcome within the group of neonates with stroke}

Amongst the neonates with stroke, absence of SEPs from at least one hemisphere $(\mathrm{n}=11)$ had a high accuracy $(90 \%)$ for unfavorable outcome: Eight of the 11 neonates developed CP, and one with concomitant severe HIE died. On the contrary, 18 of the 19 neonates with stroke but bilaterally present SEPs developed favorably, and only one developed a mild hemiparesis. In MRI, all 11 neonates with absent SEP in one or both hemispheres, including the two with favorable outcome, had an ischemic MCA stroke. On the contrary, the MRI findings in the 19 neonates with SEPs present were variable (Figs. 2B and 4).

Seizures in the EEG were more common in neonates with unfavorable than favorable outcome (Fisher's exact test, $\mathrm{p}=0.015$ ). However for outcome prediction, seizures were less accurate than SEPs (Fig. 2).

\section{Discussion}

Our data show that combining EEG and SEPs may offer unique added value in bedside testing of neonates presenting with suspected seizures or general signs of encephalopathy. In particular,

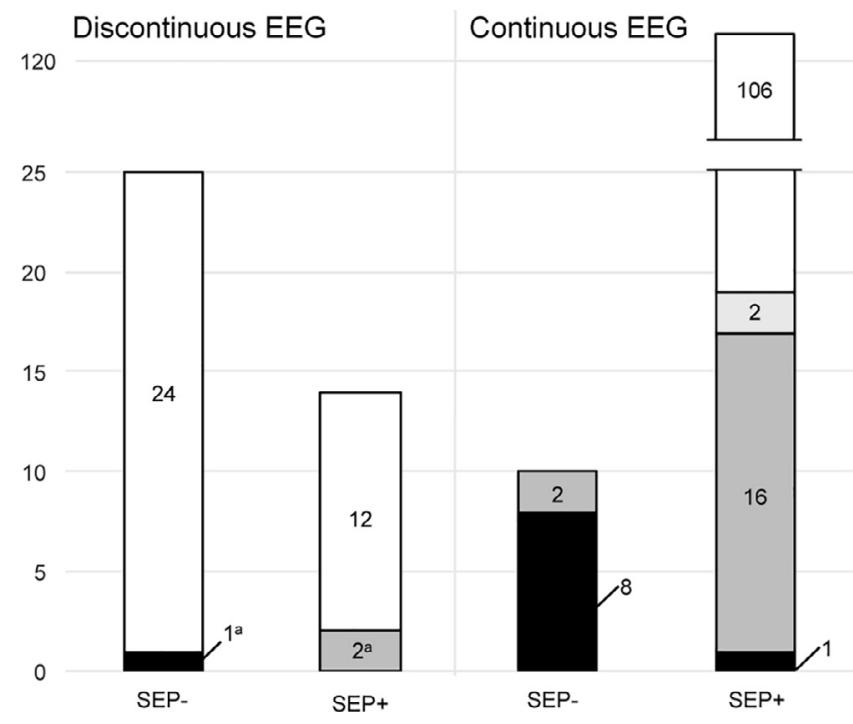

Fig. 3. Combining SEP classification with EEG background information accurately diagnoses neonates with stroke and unfavorable outcome. Black = stroke, unfavorable outcome; dark gray = stroke, favorable outcome; light gray = stroke, lost to follow-up; white $=$ not stroke. SEP- $=$ SEP absent from at least on hemisphere, SEP+ = SEP present bilaterally. Y-axis shows the number of neonates in each category, note the scale. ${ }^{a}$ stroke and concomitant HIE/asphyxia. the combination of continuous EEG background and absent SEP (uni- or bilaterally) was highly indicative of stroke with unfavorable neurodevelopmental outcome. In practice, this combination always indicated stroke and involvement of the MCA, and in $80 \%$ also subsequent development of CP. While normal SEPs did not rule out stroke, they indicated a favorable neuromotor outcome in the neonates with stroke. Our data also confirm the prior literature (Lynch et al., 2011; Low et al., 2014) in that recurrent unilateral seizures with continuous EEG background are typical of stroke, however their accuracy for outcome prediction is lower than that of SEPs.

\subsection{Challenges in early diagnosis of neonatal stroke and prediction of outcome}

Neonatal stroke is associated with significant later morbidity with, for example, $20-40 \%$ of infants with symptomatic neonatal arterial ischemic stroke (AIS) developing with CP (deVeber et al., 2000; Boardman et al., 2005; Lee et al., 2005; Husson et al., 2010; Darmency-Stamboul et al., 2012). However, detecting neonatal stroke is difficult due to the nonspecificity and oftentimes subtleness of the presenting symptoms, which calls for easily applicable bedside diagnostic methods. In addition, means for outcome prediction in the acute setting are essential for developing and studying efficacy of new neuroprotective treatment strategies, e.g. therapeutic hypothermia and erythropoietin, aiming to limit the extent of neonatal AIS and/or to promote recovery (Giraud et al., 2017).

Both ischemic and hemorrhagic neonatal stroke most commonly present with seizures within the first postnatal week (Estan and Hope, 1997; Brouwer et al., 2010; Chabrier et al., 2010; Kirton et al., 2011; Darmency-Stamboul et al., 2012; Rutherford et al., 2012; Bruno et al., 2014; Fox et al., 2016). Together they account for $19-31 \%$ of all neonatal seizures (ischemic 12-18\%, hemorrhagic 7-17\%) (Estan and Hope, 1997; Tekgul et al., 2006; Glass et al., 2016). Fewer neonates with stroke present with general signs of encephalopathy without clear clinical seizures (Estan and Hope, 1997; Brouwer et al., 2010; Chabrier et al., 2011; Moharir et al., 2011; Fox et al., 2016; Suppiej et al., 2016). Interestingly, in the present study the neonates with an unfavorable stroke outcome tended to present with recurrent apnea or general signs of encephalopathy rather than clonic motor seizures, which were the most common presenting symptom in the neonates with a favorable outcome. Hence, particularly the neonates with unfavorable stroke outcome were difficult to diagnose on clinical grounds as the characteristic focal clonic seizure manifestations were often lacking. Previously, the clonic seizure type has been shown to correlate with a more favorable outcome in a cohort including neonates with varying seizure etiologies (Mizrahi and Kellaway, 1987).

Of the neuroimaging methods cranial ultrasound, available at bedside, is sensitive in detecting hemorrhagic stroke (Mercuri et al., 1995), but it has a low sensitivity for detecting ischemic stroke within the first days after the symptoms start (Mercuri 

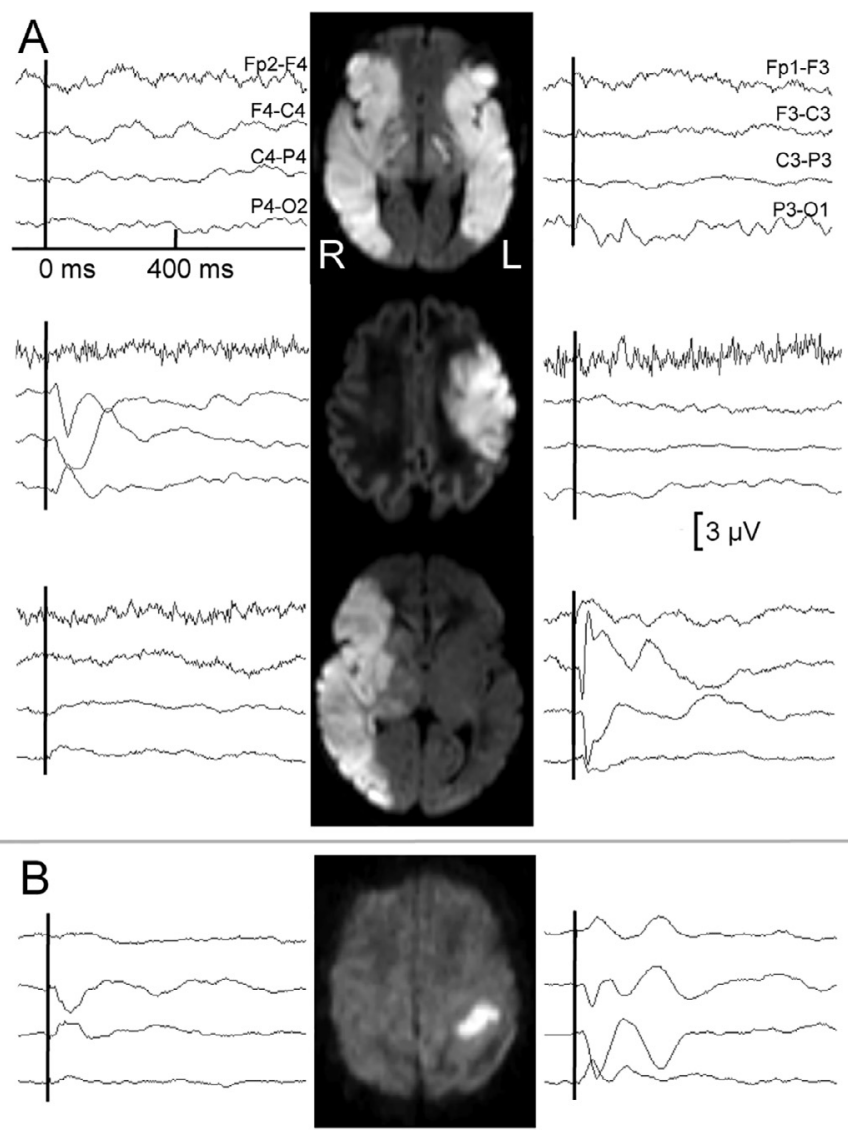
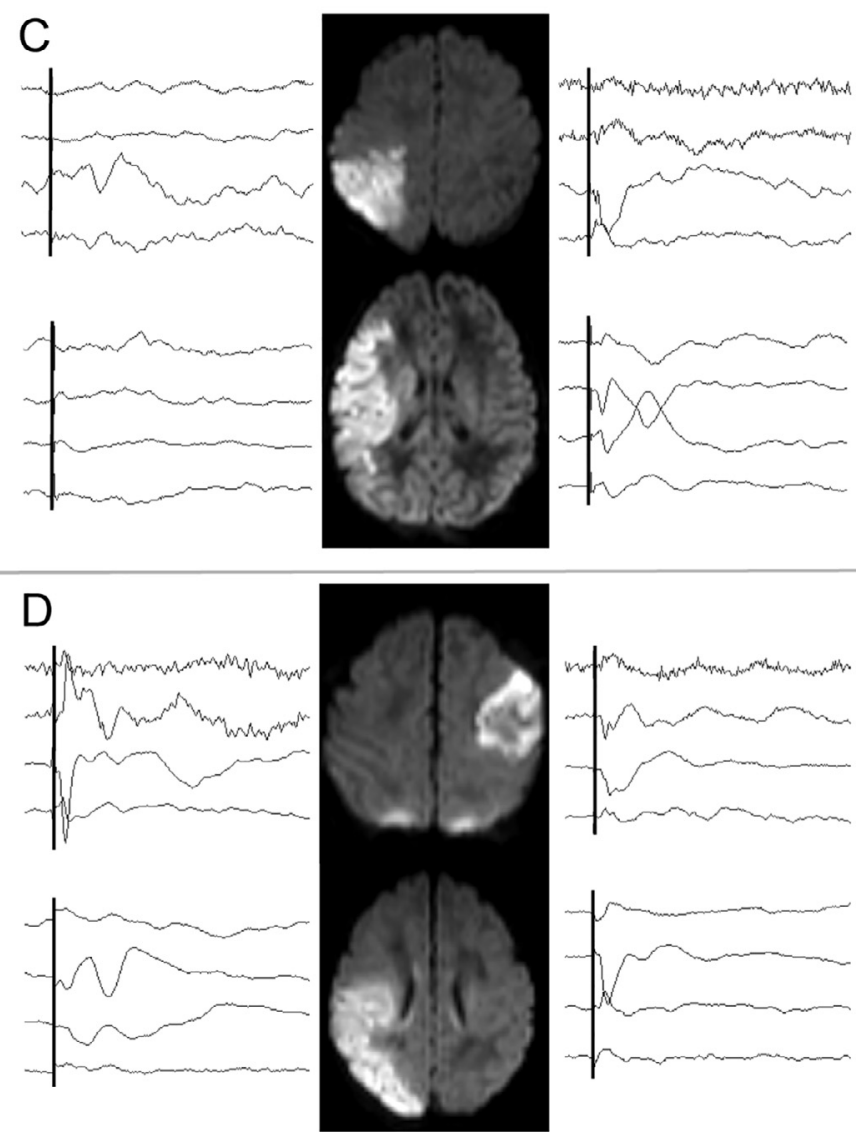

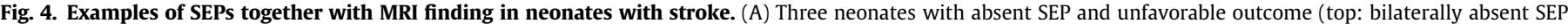

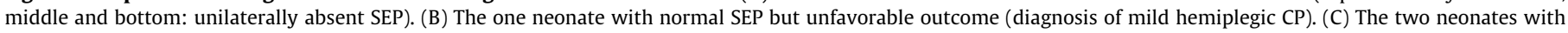
unilaterally absent SEP, but favorable outcome. (D) Two neonates with normal SEP and favorable outcome.

et al., 1995; Estan and Hope, 1997; Golomb et al., 2003). Hence, acute ischemic stroke needs to be confirmed by MRI, where diffusion weighted imaging can detect ischemic injury within hours of stroke onset (de Vries et al., 2005; van der Aa et al., 2013). MRI is also highly accurate in predicting outcome after AIS (de Vries et al., 1997; Boardman et al., 2005; de Vries et al., 2005; Kirton et al., 2008). It is, however, not available at bedside and its predictive ability is highest only after 48 hours after symptom onset (Wagenaar et al., 2017). There is little clinical or MRI data on outcome prediction after other types of neonatal stroke than AIS, but in neonates with cerebral sinovenous thrombosis the occurrence of venous infarction (deVeber et al., 2001) and other neurological comorbidity at diagnosis (Moharir et al., 2011) are associated with worse outcome.

\subsection{EEG and SEPs in the evaluation of acute neonatal stroke}

A common clinical practice considers unilateral recurrent seizures that stand out of continuous EEG activity to be suggestive of neonatal AIS (Chabrier et al., 2011). However, the evidence for this association stems from a few case series of neonatal AIS (Lynch et al., 2011; Low et al., 2014; Suppiej et al., 2016), and no information on its diagnostic specificity is available. Our present results confirm and expand this idea by showing that recurrent, unilateral seizures together with continuous EEG background are highly suggestive of stroke. However, the sensitivity of EEG-detected seizures for diagnosing stroke was low in these short-duration EEGs.

The EEG features were, however, not as accurate in stroke outcome prediction as SEPs. Our data suggest that SEPs recorded dur- ing routine EEG could serve as a bedside screening tool in neonates suspected of having a stroke. Particularly, the combination of absent SEP and continuous EEG background had a high accuracy to identify the neonates with a large MCA infarction and, consequently, a high risk for developing $\mathrm{CP}$. While there were also other etiologies (particularly HIE) underlying absent SEPs, these could be neurophysiologically distinguished from stroke based on the continuity of the EEG background, in addition to the generally different clinical setting. Although bilaterally normal median nerve EEGSEPs could not exclude stroke, they did predict good neuromotor outcome at 1-year age after neonatal stroke.

Finally, we suggest that performing SEPs simultaneously with EEG is particularly advantageous in neonates suspected of stroke, because it enables observing the spontaneous EEG during the SEP examination. Coregistration of EEG makes SEP studies more efficient and reliable as they allow selection of epochs not confounded by seizures.

\subsection{Limitations}

Our data was collected from a large and active neurophysiology unit at a tertiary level university hospital over five and half years, yet the study only includes a relatively low number of neonates with confirmed stroke. In particular, the number of neonates with non-AIS stroke was too low to draw far-reaching conclusions.

There was also some variation in the timing and length of the EEG-SEP recordings, which were affected by the constraints of clinical care. Other limitations are the relatively short follow-up period and outcome classification taken from medical records instead of a 
formal standardized neurodevelopmental test performed by an experimenter blinded to perinatal data. We do believe, however, that the follow-up information is sufficient for the robust outcome classification used in this work, since the children with stroke attended a clinical follow-up program that involves examinations by a neuropediatrician at a tertiary level hospital. Nevertheless, prospective studies using standardized follow-up protocols are necessary to confirm the results of this study.

Regarding EEG classification, we chose to focus on the most robust EEG features, which do not capture all fine EEG analysis details, e.g. hemispheric asymmetry or focal abnormalities, that have been previously reported to co-vary with some neonatal strokes (Koelfen et al., 1995; Estan and Hope, 1997; Mercuri et al., 1999; Rafay et al., 2009). In the future, a more detailed EEG analysis might gain from a quantified computational EEG analysis, which would not only be more objective but also might improve the diagnostic and prognostic accuracies from those observed in the present study.

\section{Conclusions}

Our present findings indicate a role for EEG-SEP in the early diagnostic screening of suspected neonatal stroke. We showed that the combination of a continuous EEG background and recurrent, unilateral seizures in near-term neonates was highly suggestive of stroke. Furthermore, the combination of a continuous EEG and absence of SEPs from one or both hemispheres was indicative of an MCA infarction and highly predictive of consequent development of CP. Although bilaterally normal SEPs could not exclude stroke, they did predict good short-term neuromotor outcome after neonatal stroke. EEG-SEP could, thus, serve as a bedside screening tool to direct prompt neuroimaging for those neonates with a probable MCA stroke, and consequently at highest risk of developing CP. Eventually, this work-up could lead to a faster diagnosis and open up possibilities for treatment development.

\section{Conflict of interest statement}

The authors declare no conflict of interest.

\section{Acknowledgements}

This study was financially supported by the Arvo and Lea Ylppö Foundation, Helsinki University Hospital Funds (Y920016024, Y122417013 and 1C27012974), Academy of Finland (Grant 253130), the Juselius Foundation, and Foundation for Pediatric Research. The study sponsors were not involved in the collection, analysis or interpretation of data, or in the writing of the manuscript.

\section{Appendix A. Supplementary material}

Supplementary data to this article can be found online at https://doi.org/10.1016/j.clinph.2019.02.017.

\section{References}

Boardman JP, Ganesan V, Rutherford MA, Saunders DE, Mercuri E, Cowan F. Magnetic resonance image correlates of hemiparesis after neonatal and childhood middle cerebral artery stroke. Pediatrics 2005;115(2):321-6.

Brouwer AJ, Groenendaal F, Koopman C. Nievelstein R-JA, Han SK, de Vries LS, Intracranial hemorrhage in full-term newborns: a hospital-based cohort study. Neuroradiology 2010;52(6):567-76.

Bruno CJ, Beslow LA, Witmer CM, Vossough A, Jordan LC, Zelonis S, et al. Hemorrhagic stroke in term and late preterm neonates. Arch Dis Child Fetal Neonatal Ed 2014;99(1):F48-53.

Chabrier S, Husson B, Dinomais M, Landrieu P, Tich S Nguyen The. New insights (and new interrogations) in perinatal arterial ischemic stroke. Thrombosis Res 2011;127(1):13-22.
Chabrier S, Saliba E, Nguyen The Tich S, Charollais A, Varlet M-N, Tardy B, et al. Obstetrical and neonatal characteristics vary with birthweight in a cohort of 100 term newborns with symptomatic arterial ischemic stroke. Eur J Paediatr Neurol 2010;14(3):206-13.

Darmency-Stamboul V, Chantegret C, Ferdynus C, Mejean N, Durand C, Sagot P, et al. Antenatal factors associated with perinatal arterial ischemic stroke. Stroke 2012;43(9):2307-12.

deVeber GA, MacGregor D, Curtis R, Mayank S. Neurologic outcome in survivors of childhood arterial ischemic stroke and sinovenous thrombosis. J Child Neurol 2000;15(5):316-24.

deVeber G, Andrew M, Adams C, Bjornson B, Booth F, Buckley DJ, et al. Cerebral sinovenous thrombosis in children. N Engl J Med 2001;345(6):417-23.

de Vries LS, Groenendaal F, Eken P, van Haastert IC, Rademaker KJ, Meiners LC. Infarcts in the vascular distribution of the middle cerebral artery in preterm and fullterm infants. Neuropediatrics 1997;28:88-96.

de Vries LS, Van der Grond J, Van Haastert IC, Groenendaal F. Prediction of outcome in new-born infants with arterial ischaemic stroke using diffusion-weighted magnetic resonance imaging. Neuropediatrics 2005;36(1):12-20.

Estan J, Hope P. Unilateral neonatal cerebral infarction in full term infants. Arch Dis Child 1997;76(2):F88-93.

Eytan D, Pang EW, Doesburg SM, Nenadovic V, Gavrilovic B, Laussen P, et al. Bedside functional brain imaging in critically-ill children using high-density EEG source modeling and multi-modal sensory stimulation. Neurolmage Clin 2016;12:198-211.

Fox CK, Glass HC, Sidney S, Smith SE, Fullerton HJ. Neonatal seizures triple the risk of a remote seizure after perinatal ischemic stroke Neurology 2016:86(23):2179-86.

Glass HC, Shellhaas RA, Wusthoff CJ, Chang T, Abend NS, Chu CJ, et al. Contemporary profile of seizures in neonates: a prospective cohort study. J Pediatr 2016;174:98-103.

Giraud A, Guiraut C, Chevin M, Chabrier S, Sébire G. Role of perinatal inflammation in neonatal arterial ischemic stroke. Front Neurol 2017;8:612.

Golomb MR, deVeber GA, MacGregor DL, Domi T, Whyte H, Stephens D, et al. Independent walking after neonatal arterial ischemic stroke and sinovenous thrombosis. J Child Neurol 2003;18:530-6.

Govaert P, Ramenghi L, Taal R, de Vries L, deVeber G. Diagnosis of perinatal stroke I: Definitions, differential diagnosis and registration. Acta Paediatr Int J Paediatr 2009:98(10):1556-67.

Husson B, Hertz-Pannier L, Renaud C, Allard D, Presles E, Landrieu P, et al. Motor outcomes after neonatal arterial ischemic stroke related to early MRI data in a prospective study. Pediatrics 2010;126(4):e912-8.

Kirton A, Armstrong-Wells J, Chang T, deVeber G, Rivkin MJ, Hernandez M, et al. Symptomatic neonatal arterial ischemic stroke: the international pediatric stroke study. Pediatrics 2011;128(6):e1402-10.

Kirton A, deVeber G. Advances in perinatal ischemic stroke. Pediatr Neurol 2009;40 (3):205-14.

Kirton A, deVeber G, Pontigon A-M, Macgregor D, Shroff M. Presumed perinatal ischemic stroke: Vascular classification predicts outcomes. Ann Neurol 2008;63 (4):436-43.

Koelfen W, Freund M, Varnholt V. Neonatal stroke involving the middle cerebral artery in term infants: clinical presentation, EEG and imaging studies, and outcome. Dev Med Child Neurol 1995;37:204-12.

Laugesaar R, Kolk A, Tomberg T, Metsvaht T, Lintrop M, Varendi H, et al. Acutely and retrospectively diagnosed perinatal stroke: a population-based study. Stroke 2007:38(8):2234-40.

Lee CK, Joo JY, Huh SK, Kim TS. Effects of repeated short versus single long episodes of focal ischemia on somatosensory evoked potentials and development of cerebral infarction in cats. Neurol Med Chir 1997;37:447-52.

Lee J, Croen LA, Lindan C, Nash KB, Yoshida CK, Ferriero DM, et al. Predictors of outcome in perinatal arterial stroke: a population-based study. Ann Neurol 2005;58(2):303-8.

Lee S, Mirsky DM, Beslow LA, Amlie-Lefond C, Danehy AR, Lehman L, et al. Pathways for Neuroimaging of Neonatal Stroke. Pediatr Neurol 2017;69:37-48.

Lehman LL, Rivkin MJ. Perinatal arterial ischemic stroke: presentation, risk factors, evaluation, and outcome. Pediatr Neurol 2014;51(6):760-8.

Low E, Mathieson SR, Stevenson NJ, Livingstone V, Ryan CA, Bogue CO, et al. Early postnatal EEG features of perinatal arterial ischaemic stroke with seizures. PLoS One 2014;9(7):e100973.

Lynch JK. Epidemiology and classification of perinatal stroke. Semin Fetal Neonatal Med 2009;14(5):245-9.

Lynch JK, Nelson KB. Epidemiology of perinatal stroke. Curr Opin Pediatr 2001;13 (6):499-505.

Lynch N, Low E, Rennie J, Boylan G. Comparison of seizure characteristics in full term neonates with stroke and hypoxic ischemic encephalopathy. Arch Dis Child 2011;96(Suppl 1):A39.

Mercuri E, Cowan F, Rutherford M, Acolet D, Pennock J, Dubowitz L. Ischaemic and haemorrhagic brain lesions in newborns with seizures and normal Apgar scores. Arch Dis Child 1995;73:F67-74.

Mercuri E, Rutherford M, Cowan F, Pennock J, Counsell S, Papadimitriou M, et al Early prognostic indicators of outcome in infants with neonatal cerebral infarction: a clinical, electroencephalogram, and magnetic resonance imaging study. Pediatrics 1999;103(1):39-46.

Mizrahi EM, Kellaway P. Characterization and classification of neonatal seizures. Neurology 1987;37:1837-44.

Moharir MD, Shroff M, Pontigon A-M, Askalan R, Yau I, MacGregor D, et al. A prospective outcome study of neonatal cerebral sinovenous thrombosis. J Child Neurol 2011;26(9):1137-44. 
Nevalainen P, Marchi V, Metsäranta M, Lönnqvist T, Toiviainen-Salo S, Vanhatalo S, et al. Evoked potentials recorded during routine EEG predict outcome after perinatal asphyxia. Clin Neurophysiol 2017;128(7):1337-43.

Nevalainen P, Marchi V, Metsäranta M, Lönnqvist T, Vanhatalo S, Lauronen L. Evaluation of SEPs in asphyxiated newborns using a 4-electrode aEEG brain monitoring set-up. Clin Neurophysiol Pract 2018;3:122-6.

Penchet G, Arné P, Cuny E, Monteil P, Loiseau H, Castel J-P. Use of intraoperative monitoring of somatosensory evoked potentials to prevent ischaemic stroke after surgical exclusion of middle cerebral artery aneurysms. Acta Neurochir 2007;149(4):357-64.

Rafay MF, Cortez MA, deVeber GA, Tan-Dy C, Al-Futaisi A, Yoon W, et al. Predictive value of clinical and EEG features in the diagnosis of stroke and hypoxic ischemic encephalopathy in neonates with seizures. Stroke 2009;40 (7):2402-7.

Roach ES, Golomb MR, Adams R, Biller J, Daniels S, deVeber G, et al. Management of stroke in infants and children: a scientific statement from a special writing group of the american heart association stroke council and the council on cardiovascular disease in the young. Stroke 2008;39 (9):2644-91.

Rutherford MA, Ramenghi LA, Cowan FM. Neonatal stroke. Arch Dis Child Fetal neonatal Ed 2012;97:F377-84.
Stevenson NJ, Lauronen L, Vanhatalo S. The effect of reducing EEG electrode number on the visual interpretation of the human expert for neonatal seizure detection. Clin Neurophysiol 2018;129(1):265-70.

Suppiej A, Mastrangelo M, Mastella L, Accorsi P, Grazian L, Casara G, et al. Pediatric epilepsy following neonatal seizures symptomatic of stroke. Brain Dev 2016;38 (1):27-31.

Takenouchi T, Kasdorf E, Engel M, Grunebaum A, Perlman JM. Changing pattern of perinatal brain injury in term infants in recent years. Pediatr Neurol 2012;46 (2):106-10.

Tekgul H, Gauvreau K, Soul J, Murphy L, Robertson R, Stewart J, et al. The current etiologic profile and neurodevelopmental outcome of seizures in term newborn infants. Pediatrics 2006;117(4):1270-80.

van der Aa NE, Benders MJNL, Vincken KL, Groenendaal F, de Vries LS. The course of apparent diffusion coefficient values following perinatal arterial ischemic stroke. PLoS One 2013;8(2):1-6.

van Rooij LG, de Vries LS, van Huffelen AC, Toet MC. Additional value of 2-channe amplitude integrated EEG recording in full-term infants with unilateral brain injury. Arch Dis Child Fetal Neonatal Ed 2010;95:F308.

Wagenaar N, van der Aa NE, Groenendaal F, Verhage CH, Benders MJNL, de Vries LS. MR imaging for accurate prediction of outcome after perinatal arterial ischemic stroke: Sooner not necessarily better. Eur J Paediatr Neurol 2017;21(4):666-70. 